\title{
O transtorno da compulsão alimentar (TCA) tem impacto no reganho de peso após a cirurgia bariátrica? Relato de caso
}

\author{
The binge eating disorder (BED) could influence the \\ weight regain after bariatric surgery? Case report
}

Maria Francisca F. P. Mauro', José Carlos Appolinario², Marcelo Papelbaum³, Marco Antônio Alves Brasil', João Regis Ivar Carneiro 5

\section{RESUMO}

A cirurgia bariátrica (CB) é considerada o tratamento mais eficaz para obesidade grave em longo prazo. Apesar de estar associada à resolução ou melhora das comorbidades clínicas, um desfecho possível é o reganho de peso. Um conjunto de evidências aponta a presença do transtorno da compulsão alimentar (TCA) como um dos fatores de risco associados ao reganho de peso pós-operatório. O objetivo desta apresentação de casos clínicos é discutir o possível impacto dos episódios de compulsão alimentar em pacientes submetidos à CB. Serão apresentados os seguintes casos (1): uma mulher de 41 anos, avaliada após 8 anos da cirurgia, apresentando um reganho de $22,9 \mathrm{~kg}$ e psicopatologia alimentar compatível com TCA; (2): um homem de 48 anos, avaliado no pós-operatório de 7 anos, com um reganho de $30 \mathrm{~kg}$ e exibindo queixas de beliscamento alimentar, porém sem sintomas compatíveis com TCA; (3): uma mulher de 44 anos, avaliada no pós-operatório de 3 anos, mantendo peso está-

\section{Palavras-chave}

Cirurgia bariátrica, transtornos alimentares, transtorno da compulsão alimentar, reganho de peso. vel sem reganho e que exibia à avaliação TCA. Os autores discutem, a partir destes três casos, as evidências relacionadas ao impacto da compulsão alimentar no resultado da CB. Apesar de não haver, no momento, um consenso definitivo quanto ao real impacto dos transtornos alimentares neste recrudescimento ponderal, fica claro que o clínico deve estar atento ao TCA e sua possível associação com o reganho de peso.

\begin{abstract}
Bariatric surgery (BS) is the most effectiveness long term treatment to severe obesity. However being associated with resolution or improvement of clinic comorbidities, one possible outcome is weight regain. A group of evidences appoint to the presence of BED (binge eating disorder) as one risk factor associated to post-surgery weight regain. The aim of this case report is to discuss a possible impact of binge eating in patients submitted to BS. The following cases will be presented: (1): 41 years woman, evaluated after 8 years post-surgery, showing weight regain of $22.9 \mathrm{~kg}$ and compatible eating psychopathology with BED; (2): 48 years men,
\end{abstract}

1 Universidade Federal do Rio de Janeiro (UFRJ), Instituto de Psiquiatria (IPUB), Programa de Pós-Graduação. UFRJ, Hospital Universitário Clementino Fraga Filho (HUCFF), Programa de Cirurgia Bariátrica (PROCIBA).

2 Universidade Federal do Rio de Janeiro (UFRJ), Instituto de Psiquiatria (IPUB), Grupo de Obesidade e Transtornos Alimentares (GOTA). Instituto Estadual de Diabetes e Endocrinologia do Rio de Janeiro (IEDE-RJ).

3 Universidade Federal do Rio de Janeiro (UFRJ), Instituto de Psiquiatria (IPUB).

4 Universidade Federal do Rio de Janeiro (UFRJ), Hospital Universitário Clementino Fraga Filho (HUCFF).

$14 / 3 / 2017$

Aprovado em

20/9/2017
Universidade do Estado do Rio de Janeiro (UERJ). Universidade Federal do Rio de Janeiro (UFRJ), Programa de Obesidade e Cirurgia Bariátrica (PROCIBA), Hospital Universitário Clementino Fraga Filho (HUCFF). 


\section{Keywords}

Bariatric surgery, eating disorders, binge eating disorder, weight regain. evaluated 7 years post-surgery, with weight regain of $30 \mathrm{~kg}$ and showing grazing complaints, although without compatible BED symptomatology; (3): 44 years woman, evaluated 3 years post-surgery, maintaining stable weight without weight regain and BED exhibited in evaluation. The authors discuss, from these three index cases, the associated evidences related to the impact of the binge eating in the BS result. Although, for the moment, any definitely consensus on the real impact of eating disorders in weight recrudescence is not possible, it is clear that the clinician need to be alert to BED and possible association with weight regain.

\section{INTRODUÇÃO}

A cirurgia bariátrica (CB) é a intervenção mais eficaz para o tratamento da obesidade grave em longo prazo. Além da perda de peso, acarreta melhora ou resolução das comorbidades clínicas à obesidade, tais como hipertensão, apneia do sono e diabetes'. Nos últimos 10 anos, houve um aumento de 300\% no número de procedimentos no Brasil de acordo com a Sociedade Brasileira de Cirurgia Bariátrica e Metabóli$\mathrm{Ca}^{2}$. Os procedimentos cirúrgicos empregados no tratamento da obesidade severa não são isentos de risco ${ }^{3}$. Complicações agudas (fístulas, embolia pulmonar) e de longo prazo (carências nutricionais, hipoglicemia) podem ocorrer ${ }^{3}$. Uma das possíveis complicações pós-operatórias tardias é o reganho de peso ${ }^{4}$.

O reganho de peso na CB é definido como o aumento ponderal que pode ocorrer a partir do menor peso pós-cirúrgico ${ }^{5}$. A perda de peso ocorre de forma rápida no pós-operatório imediato, em função dos mecanismos de proteção metabólico e adaptação do paciente à restrição gástrica e ao aumento inicial da saciedade ${ }^{5}$. A diminuição ponderal, no entanto, tende a se estabilizar ao longo dos primeiros 12 meses $^{4}$. Um reganho de peso de 5 a 10\% é esperado a partir de 18 meses, mas o reganho acima de 10\% é considerado por alguns autores como clinicamente significativo ${ }^{3-6}$. O transtorno da compulsão alimentar (TCA) é uma das influências a ser considerada ${ }^{1,4}$.

OTCA é o transtorno alimentar mais frequente e foi incluído na classificação diagnóstica americana (DSM-5)'. É caracterizado por episódios de compulsão alimentar, que consiste em comer, descontroladamente e em um curto intervalo de tempo, uma quantidade de comida superior a normal. Episódios ocorrem com uma frequência mínima de uma vez por semana nos últimos três meses'. Os achados na literatura divergem em relação ao real impacto clínico do TCA na perda ou manutenção ponderal após a CB ${ }^{1,-9-9}$. No pós-operatório podem ocorrer outras alterações do comportamento alimentar, como o beliscamento (grazing) ${ }^{8,10}$, que consiste em comer pequenas porções de comida fora das refeições e episódios de compulsão subjetiva em que ocorre perda de controle, sem excesso na quantidade ${ }^{1,8}$. Kalarchian et al."1', ao avaliar de forma transversal 96 pacientes em pós-cirúrgico de dois a sete anos, obteve como resultado o maior reganho de peso no grupo com TCA. Em contrapartida, Conceição et al. ${ }^{9}$, ao avaliarem transversalmente 127 pacientes com pós-cirúrgico acima de dois anos, não evidenciaram impacto do TCA no reganho de peso.

O objetivo deste artigo é apresentar três casos clínicos de indivíduos que manifestaram diferentes desfechos relacionados ao peso corporal no pós-operatório tardio da CB e discutir a possível influência do TCA no reganho de peso pós-operatório.

\section{RELATO DOS CASOS}

Os casos selecionados são do Programa de Obesidade e Cirurgia Bariátrica (PROCIBA) do Hospital Universitário Clementino Fraga Filho (HUCFF) da Universidade Federal do Rio de Janeiro (UFRJ). Os pacientes aceitaram participar deste estudo e assinaram o Termo de Consentimento Livre e Esclarecido. A seleção ocorreu de acordo com os critérios de tempo cirúrgico superior a dois anos, a alteração do comportamento alimentar e o desfecho do peso. A entrevista clínica foi realizada por psiquiatra, de forma estruturada, com os critérios da DSM-5. Os dados complementares foram estabelecidos por meio do prontuário médico.

O tempo mínimo estabelecido de tratamento clínico para obesidade foi de dois anos, com o uso de agentes antiobesidade e orientações quanto à mudança de estilo de vida (exercícios e alimentação). Posteriormente, os pacientes sem perda de peso adequada e controle das doenças clínicas associadas foram encaminhados para tratamento cirúrgico.

\section{Caso 1}

A. é uma mulher de 41 anos, casada, sem filhos, com ganho de peso a partir de seu casamento aos 20 anos. Evoluiu rapidamente para obesidade grau III (IMC $\left.\geq 40 \mathrm{~kg} / \mathrm{m}^{2}\right)^{3}$ desenvolvendo diabetes tipo 2 (DM2) e hipertensão arterial sistêmica (HAS), com indicação para CB há 10 anos.

Submetida à CB há 8 anos, apresentava no pré-operatório $162 \mathrm{~kg}\left(\mathrm{IMC}=62,54 \mathrm{~kg} / \mathrm{m}^{2}\right)$. Por meio da cirurgia, a paciente apresentou resolução das comorbidades clínicas associadas e atingiu o menor peso pós-operatório de 95 kg em 36 meses. Entretanto, com 48 meses de pós-operatório, apresentou recidiva do peso, atingindo $117,9 \mathrm{~kg}$. Foi necessário o retorno ao uso de anti-hipertensivo. 
Seu padrão alimentar é caracterizado por episódios de compulsão alimentar com frequência de três vezes na semana, configurando um TCA leve (frequência de até quatro episódios por semana). O comportamento alimentar e reganho de peso geram sofrimento psíquico na paciente, com consequente prejuízo para as suas atividades cotidianas.

\section{Caso 2}

B. é um homem de 48 anos, divorciado, taxista, que faz referência ao seu início de sobrepeso na adolescência. Aos 18 anos apresentava obesidade grau III, que evoluiu com HAS grave e dificuldades de locomoção corporal. Essa condição associou-se a prejuízo para todas suas atividades de vida diária, inclusive para trabalhar. No encaminhamento ao PROClBA, apresentava $230 \mathrm{~kg}$.

Há sete anos foi realizada a CB. Apresentou no pré-operatório (3 anos) perda de 42 kg, com peso na cirurgia de 188 $\mathrm{kg}\left(\mathrm{IMC}=50 \mathrm{~kg} / \mathrm{m}^{2}\right)$. O menor peso pós-cirúrgico ocorreu aos 24 meses $(132 \mathrm{~kg})$. Nesse período, apresentou resolução das doenças clínicas e melhora para realizar suas atividades diárias. Após 24 meses, gradualmente atingiu $162 \mathrm{~kg}$.

Em avaliação atual não apresenta TCA e belisca principalmente durante a madrugada, trabalhando. Seu beliscamento consiste em comer de forma contínua, fora das refeições, sem perda de controle ou culpa associada ${ }^{9}$. O reganho de peso não está associado a nenhum sofrimento psíquico e é atribuído ao abandono do programa nutricional.

\section{Caso 3}

C. é uma mulher de 44 anos, casada, do lar, dois filhos, que aos 24 anos iniciou seu ganho de peso. Rapidamente atingiu obesidade grau III com complicações (DM 2, HAS e apneia do sono). Encaminhada ao PROCIBA, permaneceu 1 ano em pré-operatório.

Foi submetida à CB há três anos, com peso pré-cirúrgico de $156 \mathrm{~kg}\left(\mathrm{IMC}=53,97 \mathrm{~kg} / \mathrm{m}^{2}\right)$, sem perda de peso pré-operatório. O menor peso pós-cirúrgico foi aos 12 meses (94 kg). Atualmente está com $95 \mathrm{~kg}$. Obteve sucesso cirúrgico, com resolução das comorbidades e satisfação com o resultado.

Apresenta TCA leve, com episódios de compulsão (duas vezes por semana) de forma subjetiva (a quantidade de comida é pequena, mas acompanhada da perda de controle). Não apresentou reganho de peso, mas o TCA confere sofrimento psíquico e prejuízo à sua vida social.

A Tabela 1 apresenta a evolução ponderal associada aos parâmetros clínicos.

\section{DISCUSSÃO}

A evolução de pacientes com obesidade severa para indicação de CB é cada vez mais frequente ${ }^{3}$. Os três casos apresentados caracterizam pacientes submetidos à $\mathrm{CB}$, com perda de peso inicial adequada, posteriormente evoluindo com alteração do comportamento alimentar (TCA ou beliscamento) e dois casos com reganho de peso.

Os casos 1 e 3 retratam pacientes com TCA leve, sendo o primeiro com episódios de compulsão objetivos e o segundo com episódios de compulsão subjetivos. No caso 1 ocorreu reganho de peso após 48 meses, com impacto na saúde física e retorno ao uso de anti-hipertensivo. O caso 3, sem reganho de peso, com tempo cirúrgico inferior aos demais (três anos), foi incluído para caracterizar a importância da perda de controle alimentar?. As pacientes foram submetidas a técnicas cirúrgicas distintas (Bypass e Sleeve), as quais apresentam evolução de peso distintas, devido aos mecanismos hormonais e de saciedade envolvidos, com maior reganho no Sleeve ${ }^{12}$. Os casos apresentam desfecho distinto, com evolução ponderal pior no Bypass, com diferentes tempos cirúrgicos. Essa variável também pode influenciar o reganho de peso ${ }^{13}$. Quanto ao comportamento alimentar pós-operatório, a presença de TCA é apresentada como um possível fator de impacto para o desfecho do peso ${ }^{1,7}$.

O caso 2 descreve um paciente que apresentou no pré-operatório uma perda significativa de peso (18,26\%), discutida na literatura como um fator de influência positivo para o sucesso cirúrgico ${ }^{13}$. Na evolução, aos 24 meses, com beliscamento (grazing) ${ }^{14}$ apresentou reganho de peso. Kofman et al. ${ }^{6}$, em uma amostra de 497 entrevistados pós-cirúrgico (três a sete anos), associaram recidiva de peso ao beliscamento.

Nos casos apresentados não foi descrito o padrão de comportamento alimentar pré-operatório, pois a entrevista psiquiátrica relatada neste artigo ocorreu no pós-operatório. Alguns autores sugerem aumento do risco de sintomas alimentares pós-operatório em pacientes com alterações alimentares no pré-operatório ${ }^{6}$. Não existe um consenso na literatura em relação aos critérios para reganho de peso pós-operatório, tais como pontos de corte distintos e uso de peso absoluto versus IMC, o que dificulta a comparação entre os estudos que investigaram a associação entre TCA e perda de peso pós-operatória $^{1,4,7}$. Outras limitações metodológicas incluem a dificuldade de se avaliar um transtorno alimentar com as alterações anatômicas da CB e o uso de técnicas cirúrgicas distintas'. Além disso, como já discutido, o tempo de acompanhamento pós-

Tabela 1. Evolução ponderal associada aos parâmetros clínicos

\begin{tabular}{lccccccc}
\hline & Peso na cirurgia $(\mathbf{k g})$ & Menor peso pós-cirurgia $(\mathbf{k g})$ & Reganho de peso $\mathbf{k g}(\%)$ & Tempo de cirurgia (anos) & TCA pós-cirúrgico & Técnica cirúrgica \\
\hline Caso 1 & 162 & 95 & $22,9(24,10)$ & 8 & Presente & Bypass gástrico* $^{*}$ \\
Cas0 2 & 188 & 132 & $30(22,72)$ & 7 & Ausente & Bypass gástrico $^{*}$ \\
Cas0 3 & 156 & 94 & $1(1,06)$ & 3 & Presente & Sleeve ${ }^{* *}$ \\
\hline
\end{tabular}

* Cirurgia do tipo mista restritiva e disabsortiva; *** Cirurgia do tipo restritiva. 
-operatório é importante na investigação da associação entre a presença de TCA e reganho de peso, com estudos de avaliação de longo prazo apresentando evidências mais consistentes ${ }^{10,12,15}$. De fato, os três casos apresentados exemplificam algumas das diversas variáveis clínicas e psicopatológicas que influenciam a manutenção de peso após a CB.

\section{CONCLUSÕES}

O TCA pós-cirúrgico é um diagnóstico prevalente nessa população. A avaliação desse e de outros fenômenos do comportamento alimentar e o impacto no paciente submetido à CB auxiliam o clínico em sua prática cotidiana.

\section{CONTRIBUIÇÕES INDIVIDUAIS}

Maria Francisca F. P. Mauro - Foi responsável pela escrita e revisão.

José Carlos Appolinario - Foi responsável pela supervisão e revisão.

Marcelo Papelbaum - Foi responsável pela supervisão e revisão.

Marco Antônio Alves Brasil - Foi responsável pela revisão.

João Regis Ivar Carneiro - Foi responsável pela revisão.

\section{CONFLITOS DE INTERESSE}

Não há conflitos de interesse a serem declarados.

\section{AGRADECIMENTOS}

Ao Grupo de Obesidade e Transtornos Alimentares (GOTA), Instituto de Psiquiatria (IPUB) da Universidade Federal do Rio de Janeiro (UFRJ), Programa de Cirurgia Bariátrica (PROCIBA).

\section{REFERÊNCIAS}

1. Meany $G$, Conceição $E$, Mitchell JE. Binge eating, binge eating disorder and loss of control eating: effects on weight outcomes after bariatric surgery. Eur Eat Disord Rev. 2014;22(2):87-91.

2. Sociedade Brasileira de Cirurgia Bariátrica e Metabólica (SBCBM). Disponível em: http:// www.sbcbm.org.br/wordpress/pagina-exemplo/historia-da-cirurgia-bariatrica/. Acesso em: 14 ago. 2017.

3. Mechanick JI, Youdim A, Jones DB, Timothy Garvey W, Hurley DL, Molly McMahon M, et al. Clinical practice guidelines for the perioperative nutritional, metabolic, and nonsurgical support of the bariatric surgery patient--2013 update: cosponsored by American Association of Clinical Endocrinologists, the Obesity Society, and American Society for Metabolic \& Bariatric Surgery. Surg Obes Relat Dis. 2013;9(2):159-91.

4. Odom J, Zalesin KC, Washington TL, Miller WW, Hakmeh B, Zaremba DL, et al. Behavioral predictors of weight regain after bariatric surgery. Obes Surg. 2010;20(3):349-56.

5. Heymsfield SB, Wadden TA. Mechanisms, pathophysiology, and management of obesity. N Engl J Med. 2017;376(3):254-66.

6. Kofman MD, Lent MR, Swencionis C. Maladaptive eating patterns, quality of life, and weight outcomes following gastric bypass: results of an Internet survey. Obesity (Silver Spring). 2010;18(10):1938-43.

7. Niego SH, Kofman MD, Weiss JJ, Geliebter A. Binge eating in the bariatric surgery population: a review of the literature. Int J Eat Disord. 2007:40(4):349-59.

8. Conceicao E, Vaz A, Bastos AP, Ramos A, Machado P. The development of eating disorders after bariatric surgery. Eat Disord. 2013;21(3):275-82.

9. Conceição E, Bastos AP, Brandão I, Vaz AR, Ramalho S, Arrojado F, et al. Loss of control eating and weight outcomes after bariatric surgery: a study with a Portuguese sample. Eat Weight Disord. 2014;19(1):103-9.

10. 10. Conceição EM, Mitchell JE, Engel SG, Machado PP, Lancaster K, Wonderlich SA. What is "grazing"? Reviewing its definition, frequency, clinical characteristics, and impact on bariatric surgery outcomes, and proposing a standardized definition. Surg Obes Relat Dis. 2014;10(5):973-82.

11. Kalarchian MA, Marcus MD, Wilson GT, Labouvie EW, Brolin RE, LaMarca LB. Binge eating among gastric bypass patients at long-term follow-up. Obes Surg. 2002;12(2):270-5.

12. Jammu GS, Sharma R. A 7-Year Clinical Audit of 1107 Cases Comparing Sleeve Gastrectomy, Roux-En-Y Gastric Bypass, and Mini-Gastric Bypass, to Determine an Effective and Safe Bariatric and Metabolic Procedure. Obes Surg. 2016;26(5):926-32.

13. Karmali S, Brar B, Shi X, Sharma AM, de Gara C, Birch DW. Weight Recidivism Post-Bariatric Surgery: A Systematic Review. Obes Surg. 2013;23(11):1922-33.

14. Nicolau J, Ayala L, Rivera R, Speranskaya A, Sanchís P, Julian X, et al. Postoperative grazing as a risk factor for negative outcomes after bariatric surgery. Eat Behav. 2015;18:147-50.

15. Dawes AJ, Maggard-Gibbons M, Maher AR, Booth MJ, Miake-Lye I, Beroes JM, et al. Mental Health Conditions Among Patients Seeking and Undergoing Bariatric Surgery: A Metaanalysis. JAMA. 2016;315(2):150-63. 